\title{
Mechanical properties evaluation and ultrasound diagnosis of unsaturated polyester-glass pipes
}

\author{
Gabriel Wróbel, Maciej Rojek, Małgorzata Szymiczek* \\ Silesian University Technology in Gliwice, Faculty of Materials Engineering and Biomaterials \\ *Corresponding author: e-mail: Malgorzata.Szymiczek@polsl.pl
}

\begin{abstract}
The purpose of the present work was the elaboration of research methodology of the exhaustion degree of performance properties of pipes based on unsaturated polyester and glass fibers, which were manufactured by the cross winding method on a poly(vinyl chloride) core. Within the frame of the work we conducted fatigue-ageing tests being the simulation tool of the degradation process of polyester-glass pipes. Diagnosis of the composite material condition was conducted with the use of nondestructive ultrasonic testing with the application of the echo method, in which the transition time of ultrasonic wave was determined as the identifying parameter. The registered transition time of ultrasonic wave allowed the identification of the material condition during the course of pipes exploitation.
\end{abstract}

Keywords: glass-polyester composites, ultrasound diagnostics, mechanical properties.

\section{INTRODUCTION}

One of the most rapidly developing ways of using polymeric materials concerns pipe systems. The main field of application is in the chemical, mining and gas industry, as well as plumbing works. Depending on the application, pipes are made of thermoplastic materials and composites with the warp made from unsaturated polyester and epoxy resins. These pipes must have specific properties which are determined, first of all by the conditions in which they are to be exploited as well as occupational health and safety. The growing demand for pipes results from the range of infrastructural projects that are conducted in the developing countries and the exchange of old systems. We witness an apparently growing demand for pipes for electrical, telecommunication, sanitary, and drainage systems.

Among the modern axial-cylindrical construction, the important place with constantly growing share belongs to pipes and containers made of fiber - reinforced polymer composites (glass and carbon). Products of this type are made with the use of the winding method, which allows to minimize defects through the full control of all technological parameters, which improves both the quality and repeatability of production. Winding method not only enables high level of automation and computer steering, but is also cost effective. The winding machines are applied which are based on three winding systems circumferential, screw-loop and planetary. The modern, numerically controlled winding machine guarantees the optimum strength properties of winding structure, taking into account stress distribution in the exploited product $^{1-11}$.

Cylindrical composite fiber - reinforced shells, are mainly applied in constructions operating under high static or dynamic load induced by pressure of transported medium. At the same time, these shells, besides already mentioned threats, are frequently exposed to other environmental factors, such as temperature, aggressive chemicals, gas or liquid penetration, radiation, etc.

The increase in operational safety with reference to composite shells might be achieved through elaborating the straightforward and reliable methods of assessment of material condition by applying non-destructive dia- gnostic methods. In this scope we observe the dynamic development of non-invasive diagnostics methods of polymer construction composites, which enable the current construction quality rating in the place of utilization. The specificity of diagnostic methods is highly diversified. The materials of that type are diagnosed with the use of ultrasonic and thermographic techniques, which allow the identification of defects on both processing and exploitation stages. In case of polymer matrix, these are the ageing changes (matrix degradation), whereas in case of fatigue loads, the changes are specific for this type of degradation, such as micro-cracks of the matrix, loss of adhesion between the fiber reinforced and the matrix, delamination etc. All these phenomena have a significant impact on the strength of the composite as well as exploitation of construction materials. The key condition are the diagnostic relationships associated closely and possibly unequivocally of material characteristics with the features of the diagnostic signal ${ }^{8,12-19}$.

The factors initiating degradation may be physical impacts (tension, heat, high energy radiation) as well as chemical ones (oxygen, aggressive media) ${ }^{19,20-22}$. Most frequently degradation processes are of synergistic nature and they result in interactions between the given stimuli. Such degradation process appears in the majority of cases in the application of hardening materials and their composites, e.g.: the impact of heat causes acceleration of fatigue degradation, internal stresses intensify thermal degradation, aggressive environment influences the acceleration of thermal degradation and or the fatigue of composites. In practice we can encounter much more complicated structure of degradation factors. For example, the polymer composites applied as parts of ships and yachts are exposed to simultaneous effects of mechanical stress, UV radiation, temperature, sea water and microorganisms. The degradation process causes changes in polymer which are most frequently irreversible, being the result of chemical reactions, such as: crosslinking, thermal oxidation, cutting chains or even destruction. Another example is the effect of cyclically variable load on a composite ${ }^{19,23}$, which is accompanied by irreversible thermo-kinetic processes. The mechanism of material deterioration in time is complex. Deterioration may take place as a result of matrix and fibres cracking, 
delamination and decohesion. The fatigue process causes the gradual degradation of composite structure, which results in reduction of stiffness. Mechanisms of polymer construction composites depend on the architecture of strengthen $^{\mathbf{8 - 1 0}}$. In the case of laminates, the destruction process runs in stages from warp micro-cracks to phase to phase dissection, which in consequence may also cause delamination.

\section{EXPERIMENTAL}

\section{Material}

The pipes samples were based on unsaturated polyester resin reinforced with $52 \%$ wt. of glass fibers type E. Composite shells were winding on the pipes of poly(vinyl chloride), which made the integral part of tested pipes. In order to ensure proper qualities, we applied three interlayers made of glass mat with weight of $450 \mathrm{~g} / \mathrm{m}^{2}$, on which were filament winding successive roving (each layer 8 passings of the support). The final layer of the beam is 16 passings of the support. The whole of the construction was secured with the mat with the weight of $130 \mathrm{~g} / \mathrm{m}^{2}$ (Fig. 1).

As the warp we applied the mixture of resins Polimal 104 TS (from OrganikaSarzyna Company, Nowa Sarzyna, Poland) and Estromal 14. CNP -03/P (From Erg-Chemical Company, Pustków, Poland). Polimal 104 TS is flame retardant orthophtalic and thixotropic unsaturated polyester resin of medium elasticity. The resin contains halogenated compounds and antimony trioxide, which allow the lowering of the time of self-extinguishing (below 5 s, oxygen index 28\%). Estromal 14.CNP-03/P is the resin of medium reactivity, of ortho/terephtalic type, of self-extinguishing properties, and increased chemical resistance properties.

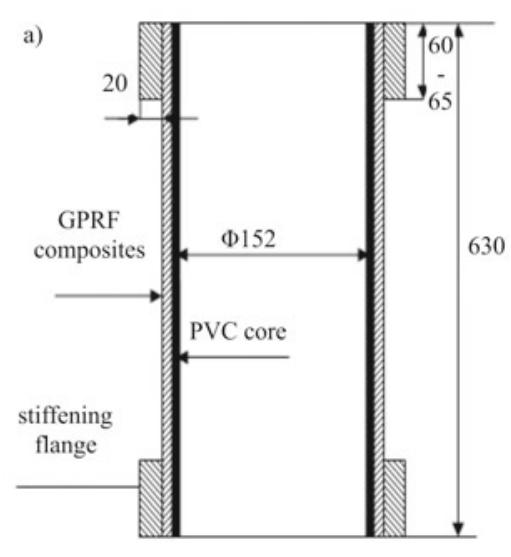

b)

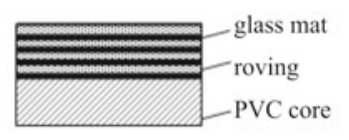

Figure 1. Test sample (a) and scheme of layers (b)

Taking into account the fact, that the pipes are intended for mining industry, soot which lowers the surface resistivity below $10^{6} \Omega$ was added to the resin. Pipe samples
(Fig. 1) with measurements: inner diameter $\varphi 152 \mathrm{~mm}$, length $630 \mathrm{~mm}$, thickness of composite layer $7 \mathrm{~mm}$, thickness of PVC pipe $4 \mathrm{~mm}$, were made with the use of a winder. Fiberglass stand composed of 16 bundles was cross winded at the angle of $54^{\circ}$. In order to stabilize the properties, all tested pipes were subjected to thermal treatment in air circulating chamber with a temperature of $60^{\circ}$ during $24 \mathrm{~h}$.

At the ends of pipe samples the flanges were made, using the method of circumferential winding, to ensure stiffness and dimensional stability in the sealed places during the fatigue tests. Between the steel core and a sample, a specially constructed gasket made of polyurethane elastomer was inserted. This sealing method of the sample resulted in the domination of the hoop stresses and the negligible radial stresses (Fig. 2). It enabled the long-term sealing of the samples connection with the core elements of the fastening assembly and guaranteed the tightness of the pipe in the conditions of variable load pressure. The steel core performed the construction function of the seal system and minimized, due to its measurements, necessary volume of hydraulic fluid (water) which is necessary to force the set pressure. A pipe without a PVC core, could lose its tightness (pipe sweating) before losing its performance properties (bearing capacity and tightness) to the degree that disqualifies it as a constructing and transmission pipe.

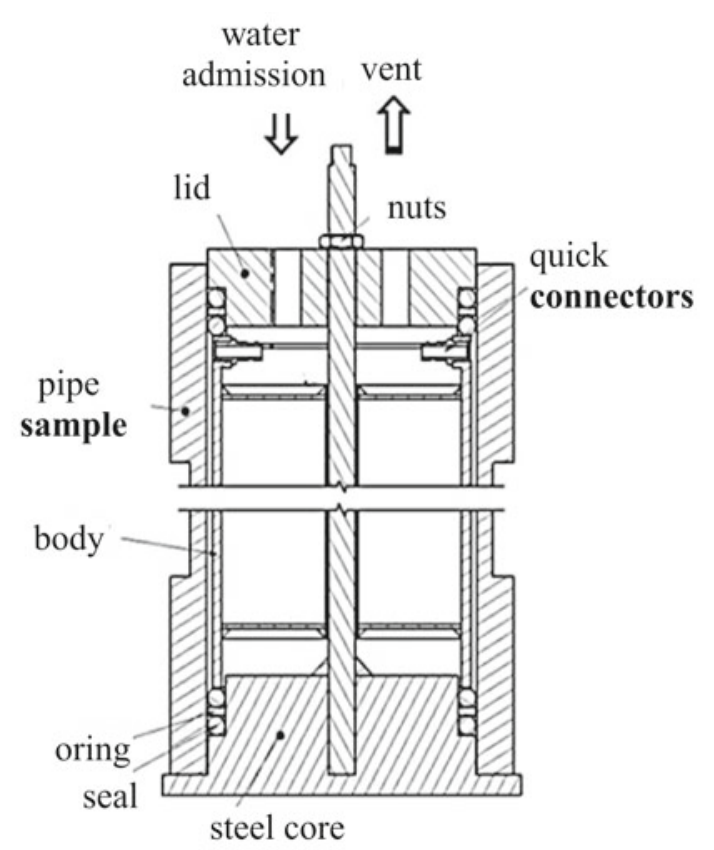

Figure 2. The sealing scheme of composite tested samples

\section{Testing}

The research was conducted with the use of five samples simultaneously, which were subjected to fatigue loads with the same load profile, at the working stations described in works ${ }^{24-26}$, at the temperature of $30^{\circ} \mathrm{C}$ for various maximum pressures - $10 \mathrm{MPa}$ and $7 \mathrm{MPa}$. We assumed the rectangular load cycle, where the time of pressure growth was $1,5 \mathrm{~s}$, with the loading time with maximum pressure of $2 \mathrm{~s}$. The test was conducted in an aqueous medium.

The pipe sample was subjected to circumferential and longitudinal strain, due to applied pressure. Taking into 
account the change in the tested pipe while loaded by applied pressure (increase in diameter and shortening of length) the sealing should be resistant to frictional wear. During the measurement, the counter records the number of load cycles and allows the program breaks for performing non-destructive testing in subsequent phases of ageing-fatigue process.

In the case of tightness loss (perforation of the sample), the switching off of the working station occurs. The research program embraces the destruction of the samples part in the test conditions. The sample marked with a grid of measurement points periodically removed from the bath and subjected to ultrasound scanning, marking the transition time of the ultrasonic wave. The changes in transition time of the ultrasonic wave was recorded always in the same measurement points. The view of working station for the ultrasound research is presented in Figure 3.

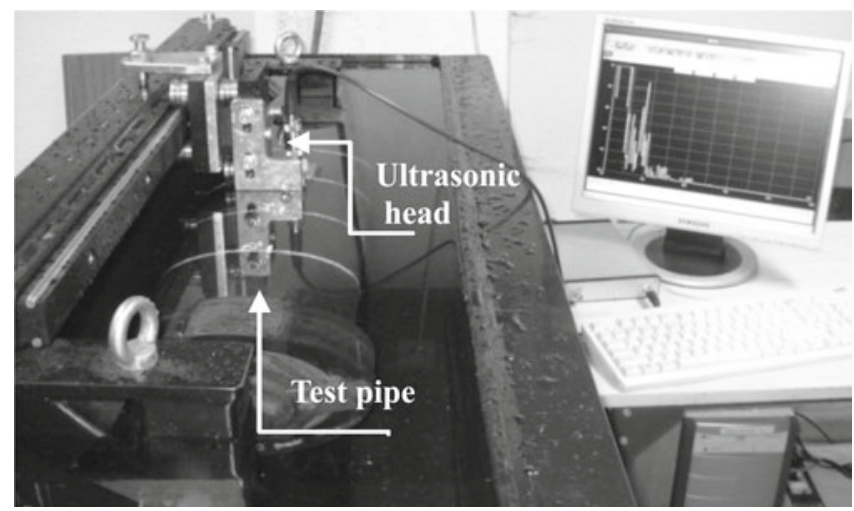

Figure 3. Working station for ultrasonic research

Within the presented work, the assessment of composite material loss of bearing capacity was conducted on the basis of non-invasive ultrasound diagnostics with the application of the echo method, in which the signal sent by the head is reflected from the opposite surface in case of material with continuous structure ${ }^{\mathbf{1 2 - 1 9}}$. In case of an appearing defect, e.g. through delamination, ultrasonic wave reflects the discontinuity. Measuring the transition time of ultrasonic wave makes it possible to locate a defect. Applying the echo method requires the access to one of the object's surfaces, which is undoubtedly a good point of the method. The echo of discontinuity appears between a starting impulse, which makes the imaging of the signal, rousing a ultrasonic transducer to oscillation, and the echo of the object's bottom or between further echoes of object's bottom. The location of discontinuity, in the shape of voids, bladders, dissections, micro-cracks is based on the measurement of the transition time of ultrasonic wave, at variable speed of a given kind of ultrasonic wave, given material and is based on the relationship ${ }^{13}$ :

$l=\frac{c t}{2}$

where: $l$ - path of the ultrasonic waves, $c$ - speed of given kind of ultrasonic wave, in given material, in $\mathrm{m} / \mathrm{s}$ or $\mathrm{mm} /$ $\mu \mathrm{s}, t-$ transition time of ultrasonic wave, in $s$ or $\mu s$. The number 2 in denominator results from the fact that the path of the wave, in the research where the echo method is applied, is twice longer than the distance between diffusive reflectors and heads. It should be pointed out, that the wave flight influences the kind, shape, surface area, distance from discontinuity head, its orientation in relation to wave input and the characteristics of the researched material ${ }^{13-19}$.

\section{Evaluation of mechanical properties}

In order to establish the changes in strength properties, we conducted the research concerning the tensile circumferential strength in accordance with the norm PN-EN $1394^{27}$ with the use of strength testing machine Fritz Heckert FPZ 100/1. View samples and working station for determining of the apparent initial circumferential tensile strength are shown in Figure 4.
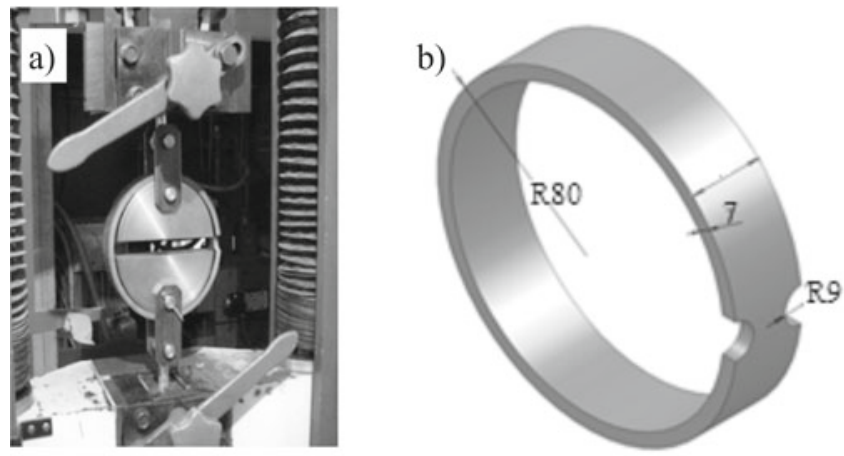

Figure 4. View of working station (a) and sample dimension (b)

The apparent initial circumferential tensile strength $\left(\sigma_{c}\right)$ was calculated from the formula:

$\sigma_{c}=\frac{F_{t}}{b \times g}$

where: $F_{t}$ - tensile force, $b$ - averaged width measurement from the outside and inside diameters, $g$ - averaged width of three measurements.

The flexural strength tests were performed according to standard PN-EN ISO $178^{28}$ on the machine Zwick / Roell Z020. The study was conducted on 5 samples cut out along the pipes. In the Figure 5 was presented view of test stand and sample dimensions. Three-point flexural test was conducted at a speed of $5 \mathrm{~mm} / \mathrm{min}$ and the flexural strength and modulus were determined.

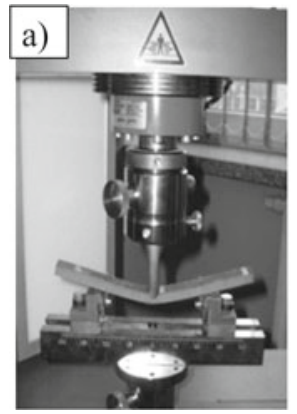

b)

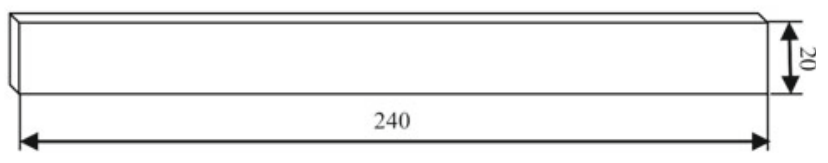

Figure 5. View of working station (a) and sample dimension (b) 


\section{Microscopic investigations}

The research concerning changes in polyester-glass composite structure was conducted with the use of the light microscope Axio Observer Z1 Zeiss. Samples were prepared from the pipes were treated ageing - fatigue degradation.

\section{RESULTS AND DISCUSSION}

The purpose of the hereby presented work was the establishment of the relationship between the ultrasonic wave transition time and performance properties of polyester-glass composites based pipes produced by winding method. The conducted test concerned the fatigue-ageing degradation, which was realized at the working station described earlier ${ }^{22-24}$. The assessment of the exhaustion degree of strength properties was carried out in two ways - with non-destructive methods (measurement of ultrasonic wave transition time) as well as the destructive ones (tests on flexural strength, tensile circumferential strength and microscopic fracture analysis). It should be pointed out that for analysis and relationship establishment, we assumed the surface in which delamination did not occur. The assessment was conducted with the use of the ultrasonic defectoscope UMT 17 equipped with the head Parametric with the frequency of 2,25 $\mathrm{MHz}$ which enables measurement in the aquatic environment.

The relationship between the ultrasonic wave time and the number of fatigue cycles for samples loaded under maximum pressure of $10 \mathrm{MPa}$ is shown in Figure 6 . The obtained results refer to single pipe samples, and the transition times presented are the average value of five measurements for given data points plotted on the external surface of the pipe. Points given are marked on the circumference of the pipe, while on the axis y there are the transition time of ultrasonic wave. Each quarter corresponds to one polyester-glass pipe. As it can be observed, during the first phase of the test, the transition time of ultrasonic wave was not subjected to any change. In the next stage, the shortening of the transition times occurred, however, we cannot specify unequivocal threshold of the number of cycles being the determinant of time decrease as it depends on the load, to which the sample is subjected during the test as well as the quality of the tested composite pipe. Together with the increase in the number of cycles, the decrease in the transition times of ultrasonic wave was observed. The shortening of the transition times of ultrasonic wave was caused by the appearance of non-continuities in the composite structure.

A distinct decrease in the transition time can be observed, which suggests the appearance of flaws on only one part of the pipe - points $1 \div 9$. However, in case of points $10-16$ we affirmed the large distribution of the data. Due to the fast destruction of the pipes at the load of $10 \mathrm{MPa}$, which did not exceed 20000 cycles, the pressure was lowered to $7 \mathrm{MPa}$, which enabled the testing time extension to 110000 cycles, which in turn allowed to observe in a periodical way the changes in the structure of the tested polyester-glass pipes.

Loading the samples by the maximum pressure of 10 MPa caused cracks in both internal covering and com-

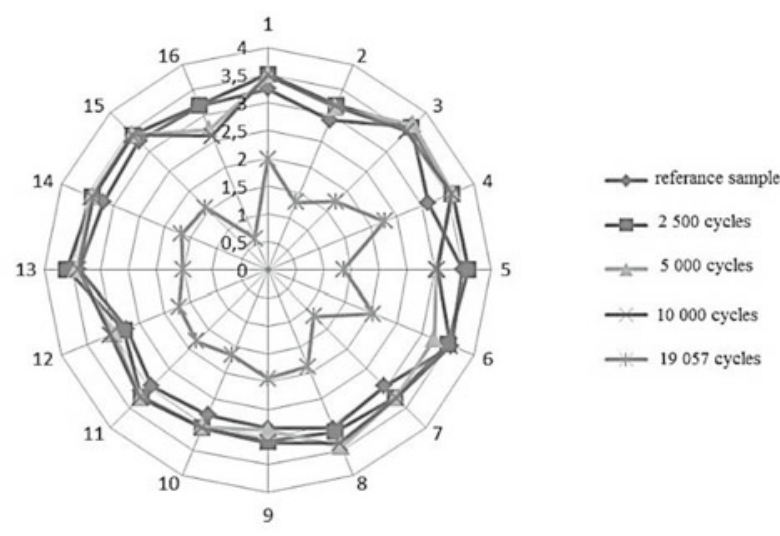

Figure 6. Relationship between the transition time of ultrasonic wave and the number of cycles for samples loaded under maximum pressure of $10 \mathrm{MPa}$

posite shell in the vicinity of point 16 , where the lowest transition time was registered - Figure 7. However, it should be pointed out that such load exceeded the working pressure of the pipe to a large extent. In case of composite constructions, the problem is large distribution of data points, which makes interpretation difficult.
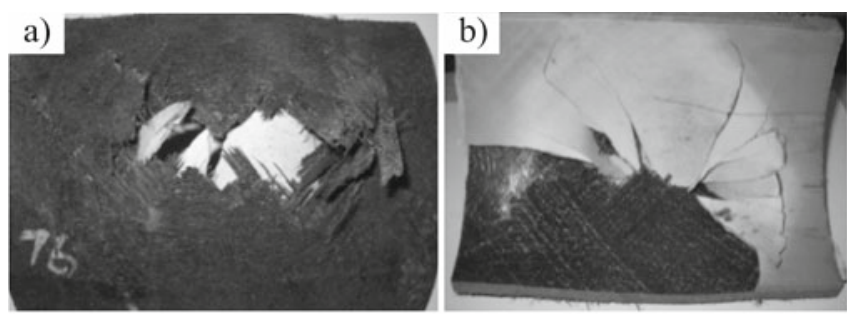

Figure 7. Image of external (a) and internal surfaces (b) destruction for samples loaded with a maximum pressure of $10 \mathrm{MPa}$

Figure 8 represents the relationship between the ultrasonic wave time and the number of fatigue cycles for samples loaded under maximum pressure of $7 \mathrm{MPa}$.

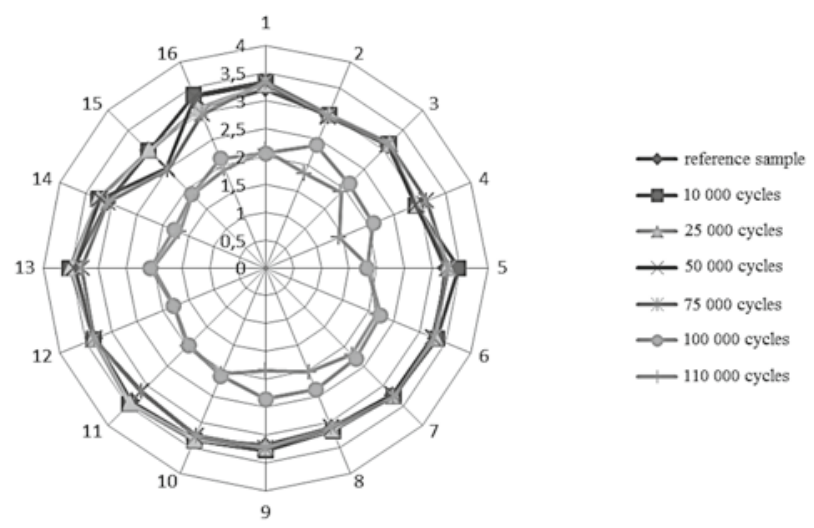

Figure 8. Relationship between the transition time of ultrasonic wave and the number of cycles for samples loaded under maximum pressure of $7 \mathrm{MPa}$

As it can be observed in the graph (Fig. 8), greatest changes of transit time ultrasonic wave occur in the point 15 and 4 . There are gradual changes related to the occurrence of micro-cracks in the first step (25 000 cycles), which later are aggravated (up to 75000 cycles), however, the material does not exhibit apparent delamination. In the final stage, will appear delamination 
(100 000-110-000 cycles). View of the destruction of pipe after 110000 cycles degraded fatigue with a maximum pressure of $7 \mathrm{MPa}$ is presented - Figure 9 .

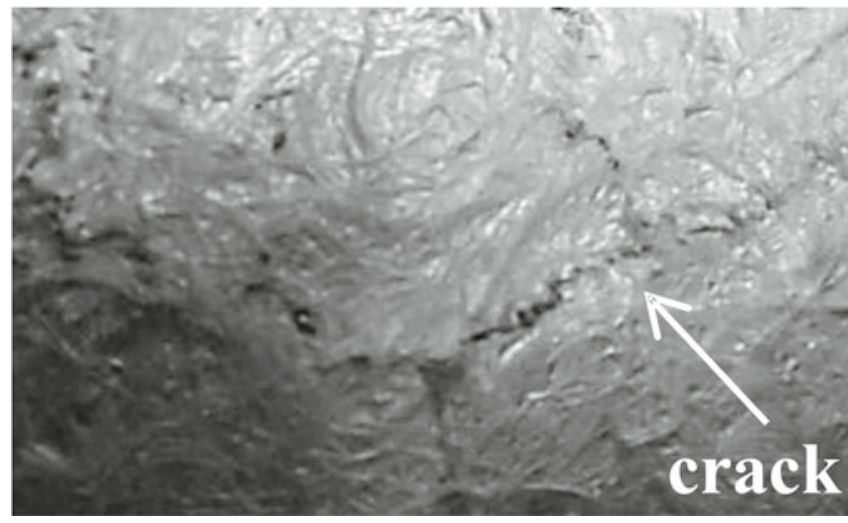

Figure 9. Image of external surfaces destruction for samples loaded with a maximum pressure of $7 \mathrm{MPa}$

This is an image observed by the destruction of the external surface of the pipe. However, there was no destruction of the inner layer of PVC, as in the case of composite loaded with maximum pressure of $10 \mathrm{MPa}$ (Fig. 7).

The evolution of the composite delamination process during the fatigue-ageing test could be easily observed in Figure 10. In fact, no cracking or delamination was observed in the initial stage (reference sample). However, the delamination of the composite is evident.

For the purpose of the investigation of the tested composite, the samples were cut out from the places where the significant shortening of the transition times of ultrasonic wave was registered, and were then subjected to an organoleptic test. On the sample section the clear delamination of the composite was observed (Fig. 10b and Fig. 10c). In the next stage the samples were subjected to an ultrasound test, which confirmed that in the points of delamination the transition time of ultrasonic wave was much shorter than in the sample which did not exhibit flaws (Fig. 10a). The test unequivocally indicated that the transition times of ultrasonic wave might be the effective method to assess the condition of glass-polyester composite during its exploitation. Figure 10 represents the variations in the ultrasonic wave transition times as a function of the number of cycles. The observed changes in ultrasonic wave transition time from the initial value of $4.03 \mu$ s (section A Fig. 10a) and $3.99 \mu$ s (section B, Fig. 10a) to the value of $1.99 \mu$ s (section A Fig. 10b) and $2.11 \mu$ s (section B, Fig. 10c) allow the diagnosis of the material. The ultrasonic wave transition time $3,81 \mu \mathrm{s}$ in section B Figure 10b suggests the occurrence of micro-defects, leading to delamination Figure 10c section B (transition time $2.11 \mu \mathrm{s}$ ). Differences in the transition time $(4.03 \mu \mathrm{s}-$ section A and $3.99 \mu \mathrm{s}-$ section B) due to variable thickness of the composite coating as a result of the manufacturing process.

The dependence of the of ultrasonic wave transition times is shown in figure 11 as a function of the number of cycles for sections A and B. We noticed a 50\% decrease of the transition time of section A after 50000 cycles, while in section B the same decrease was obtained after more than 100000 cycles.
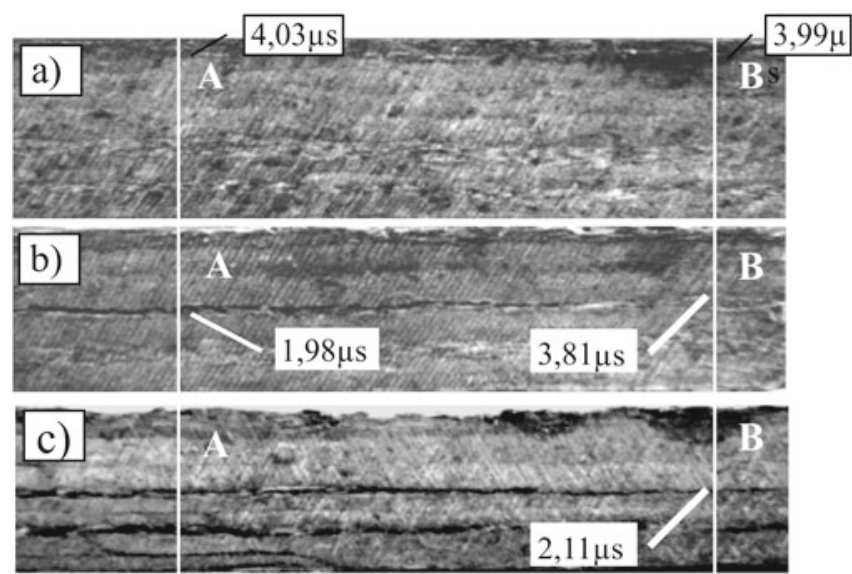

Figure 10. Photographs of composite samples after various stages of fatigue-ageing test initial state - reference sample (a), beginning of delamination (b), delamination (c)

Figures 10 and 11 are examples of observed changes that result from the ageing - fatigue degradation process.

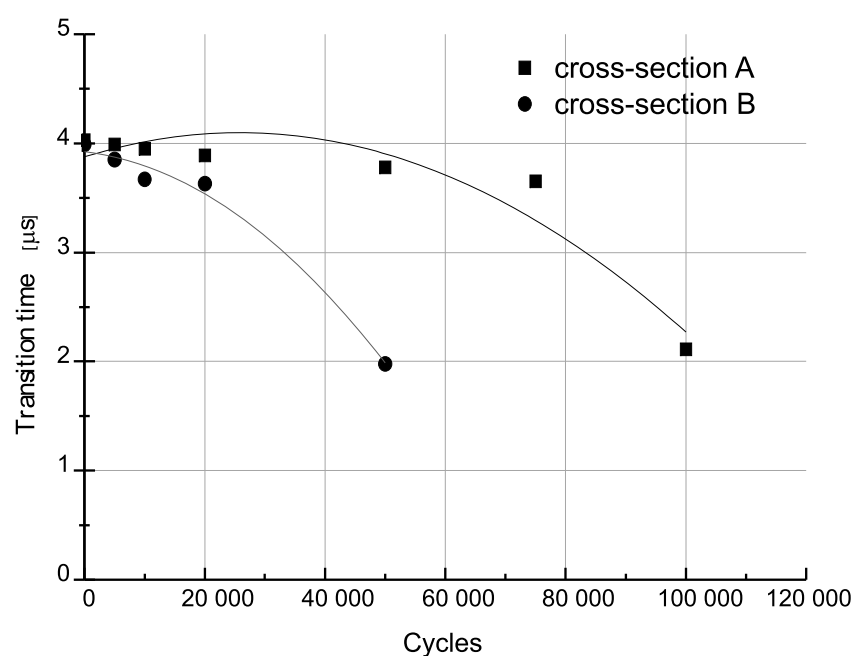

Figure 11. Dependence of the ultrasonic wave transition times on the number of cycles for sections A and B

During the test conducted on the cross-sections of the cut out samples, we observed delamination on the border between continuous filament and glass mat spacer instead of variations in the ultrasonic wave transition time. Moreover, after the mechanical rupture of the samples, we noticed the lack or too weak supersaturation of glass mat with polyester resin instead of delamination within the composite. The samples cut out from the area, where the transition time of ultrasonic wave remained unchanged during the fatigue test, showed no delamination on the border between glass mat and continuous filament. On the basis of scanning of the external surface of the pipes we specified the delamination spots. The results of the work were made available to the producer of the pipes, which allowed the changes in technology including the application of mat spacer with lower weight $\left(350 \mathrm{~g} / \mathrm{m}^{2}\right)$ and exact supersaturation in the course of winding the pipes.

The results of the tests concerning the apparent initial circumferential tensile strength as function of the number of cycles are shown in figures 12. It can be noted that both parameters values decreased with increasing number of cycles. The tensile strength decreased by approximately $45 \%$ after 100000 cycles. The decrease of the tensile 
properties might be related with the fatigue ageing of the tested materials. However, it should be pointed out

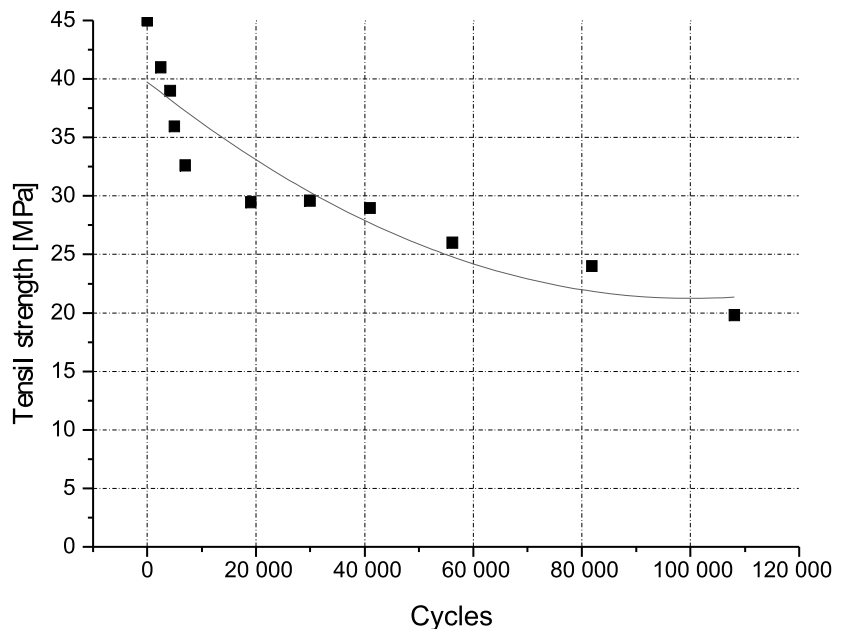

Figure 12. Dependence of the apparent circumferential tensile strength on the number of cycles

that this test is not the best measurement of changes in strength properties, as the reinforcing fibers are mainly subjected to the tensile forces.

Figures 13 and 14 show the relationship between flexural strength and modulus as function of number of

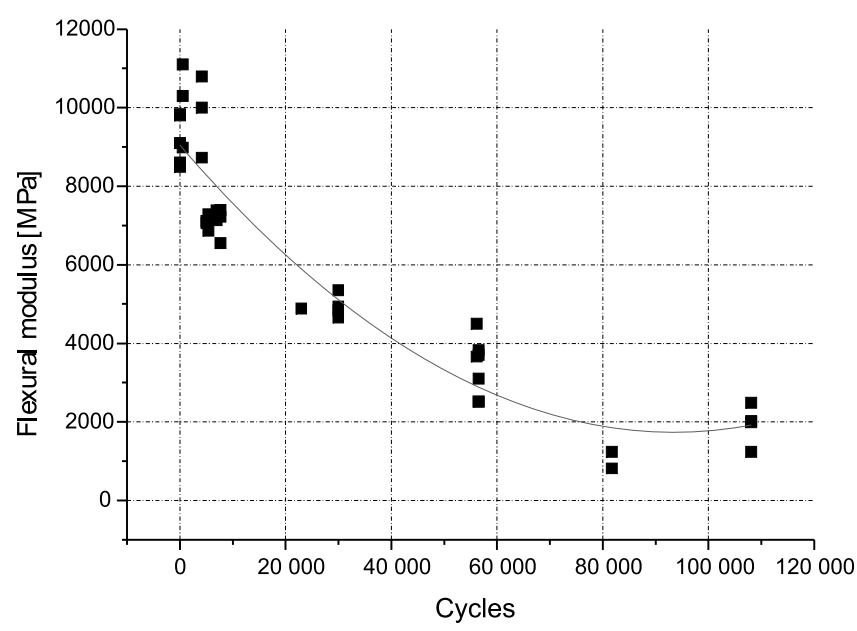

Figure 13. Dependence of the flexural modulus on the number of cycles

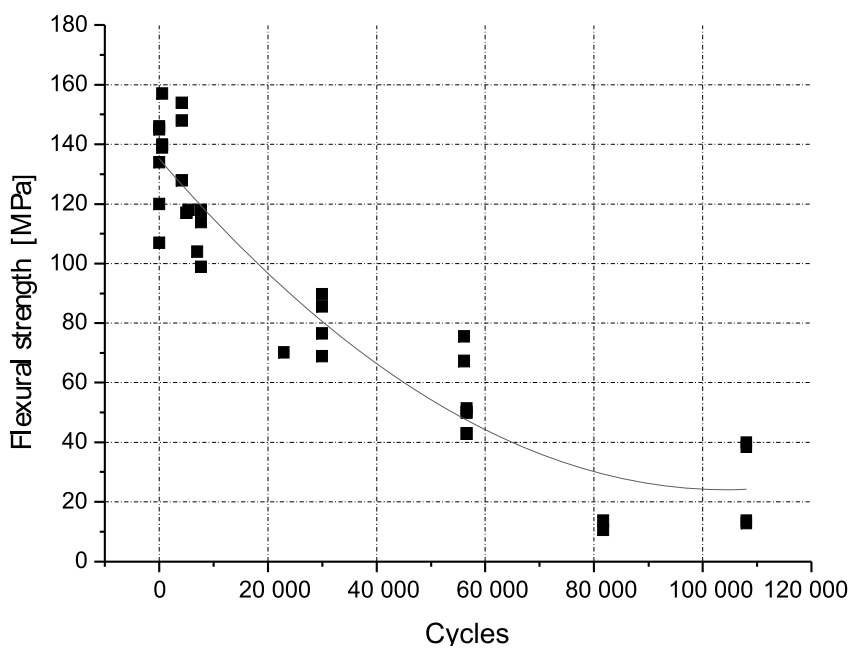

Figure 14. Dependence of the flexural strength on the number of cycles fatigue cycles. The flexural modulus decreased by more than $70 \%$ for the same fatigue test time.

As it can be observed, the strength properties fall together with the number of cycles. However, they are the situation when the delamination does not occur, which means the researched area is the material in condition shown in Fig. 10 and 11 B. Within the area A (Fig. 10 and 11) faster delamination occurs, which eliminates the usage of the composite.

Microscopic images of the changes in the material with various degree of degradation during the fatigue-ageing tests are shown in Figure 15. As it can be observed in Figure 15b, flaws appear first in the area glass mat spacers, which finally lead to delamination of the sample (Fig. 15c).
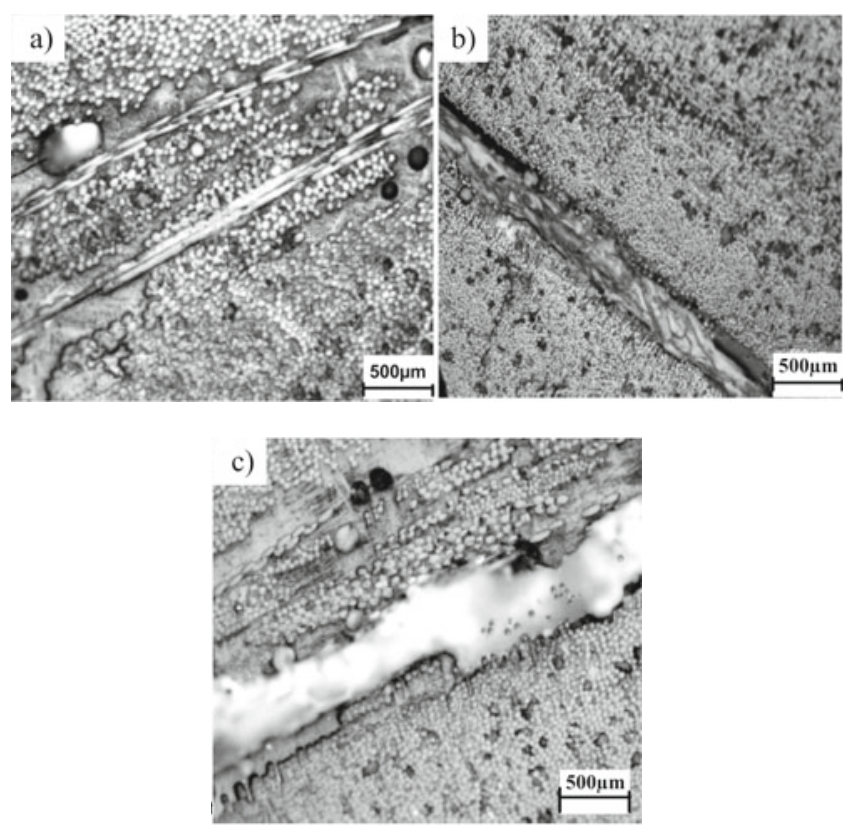

Figure 15. Exemplary microscopic images of changes that occur in material in the course of fatigue-ageing degradation a) initial state, b) after 50x103 cycles, c) after 100x103 cycles

The relationship between the ultrasonic wave transition time and flexural strength is exhibited in Figure 16. It should be noted that this relationship (Fig. 16) refers to an area where one can observe dispersed defects such as micro-cracks, but no delamination.

The developed diagnostic relationship allows for continuous monitoring and diagnosing a large approximation of the state of the material (the correlation coefficients

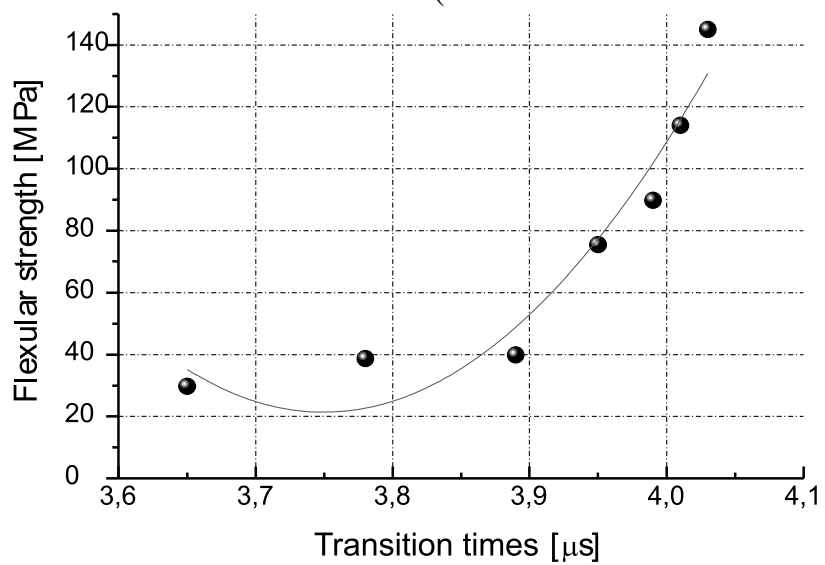

Figure 16. Relationship between flexural strength and ultrasonic wave transition times 
$\mathrm{R}^{2}$ is 0,94 ), which prevents catastrophic damage to the structure.

\section{CONCLUSIONS}

The following conclusions can be drawn from the obtained results:

The transition time of ultrasonic wave through the composite depends on the distance of the flaw from the surface of the material and it decreased with decreasing the distance of the flaw generated during the fatigue test. The transition time decreased by $50 \%$ after 50000 cycles for section A and more than 100000 cycles for section $\mathrm{B}$.

With the increase in the number of cycles are falling mechanical properties - apparent initial circumferential tensile strength, flexural strength and modulus. The largest decrease was recorded for flexural strength $-85 \%$. The smallest decrease was observed for apparent initial circumferential tensile strength $-45 \%$, which is caused by stretching of the peripheral glass fibers.

The relationship between the transition time ultrasonic wave and flexural strength, developed on the basis of test results, allows for non - invasive diagnosis of polyester-glass composite produced by filament winding.

The developed method might become the effective tool for state evaluation of polyester-glass composite during exploitation. In order to achieve more accurate analysis, the extended ultrasound research of polyester-glass pipes winded on the cores of polyvinylchloride, should be conducted.

\section{LITERATURE CITED}

1. Janson, L.E. (December, 1993). Method for tightness testing of plastics pressure pipelines. Constr. Build. Mater. 7(4), 241-244. DOI: 10.1016/0950-0618(93)90008-Z.

2. Hearn, G.L. (March 2002). Electrostatic ignition hazards arising from fuel flow in plastic pipelines. J. Loss Prevent. Proc. 15(2), 105-109. DOI:10.1016/S0950-4230(01)00061-4.

3. Marsh, G. (March 2006). Composites keep pipelines flowing. Reinf. Plast. 50(3), 34-36. DOI: 10.1016/S0034-3617(06)70940-7. 4. Jones, R.M. (1998). Mechanics of composite materials $\left(2^{\text {nd }}\right.$ ed.). Philadelphia, USA: Taylor \& Francis Press.

5. Królikowski, W., Kłosowska-Wołkowicz, Z. \& Penczek P. (1986). Resin and polyester and laminates ( $2^{\text {nd }}$ ed.). Warsaw, Poland: WNT Press. (In Polish).

6. German, J. (1996). The basis of mechanics of fibers composites: a scripts for the students of higher technical schools. Cracow, Poland: Cracow University of Technology Publishing. (In Polish). 7. Hull, D., Clyne, T.W. (1996). An introduction to composite materials. Melbourne, Australia: Cambridge University Press. 8. Błażejewski, W. (2007). The application of acoustic emission in comparative research of braid carriers of composite bottles. In Wróbel G. Polymers and composites construction. Cieszyn, Poland: Logos Press Publishing (In Polish).

9. Czulak, A. (2010). Shaping of the structure and properties of the fiber glass-woven composite pipe element, Unpublished doctoral dissertation. Technishe University of Dresden, Germany. 10. Błażejewski, W. (2009). Methodology of selection of the structure of composite braid carriers with reference to containers made by the winding method. Tech. Transactions - Mechanics z. 1-M, 9-14. (In Polish).

11. Cohen, D. (1997, July). Influence of filament winding parameters on composite vessel quality and strength, Comp.: Part A, 28A, 1035-1047. DOI: 10.1016/S1359-835X(97)00073-0.
12. Deputat, J. (1997). Non-destructive methods of research on materials properties. Warsaw, Poland: Biuro Gamma Press (In Polish).

13. Śliwiński, A. (2001). Physics for industry. Ultrasounds and their applications ( $2^{\text {nd }}$ ed). Warsaw, Poland: WNT Press (In Polish). 14. Kozień, M.S. (2005). Hybrid Method of Evaluation of Sounds Radiated by Vibrating Surface Elements, J. Theor. Appl. Mech. 43,1, 119-133 (In Polish).

15. Lewińska-Romicka, A. (2001). Non-destructive research. The basis of defectoscopy. Warsaw, Poland: WNT Press (In Polish). 16. Ostachowicz, W., Wandowski, T., Malinowski, P. (2008). Elastic Wave Phased Array for Damage Localisation, J. Theor. Appl. Mech. 46, 917-931. (In Polish).

17. Wróbel, G. (2008). Non-destructive methods of engineer research on polymer materials. Gliwice, Poland: Silesian University of Technology Publishing, (In Polish).

18. Rojek, M., Szymiczek, M., Wróbel, G. (2011). Non-destructive methods of research on polymer materials, Plast. Proces. 6(144), 507-510 (In Polish).

19. Rojek, M. (2011): Methodology of diagnostics research of layered composite materials with polymer warp. Gliwice, Poland: International OCSCO World Press (In Polish).

20. Martin, R. (2008). Ageing of composites, Cambridge, England: Woodhead Publishing Limited.

21. Bogdan-Włodek, A., Kozioł, M. \& Myalski, J. (April, 2012). Influence of surface treatment on the wetting process of jute fibres with thermosetting polyester resin. Pol. J. Chem. Tech. 14,1, 21-27. DOI: 10.2478/v10026-012-0054-9.

22. Landowski, M., Budzik, M. \& Imielińska, K. (March, 2011). On degradation of glass/polyester laminate immersed in water. Advan. Mater. Sci. 11, 1, 35-39. DOI: 10.2478/v10077-011-004-x. 23. Harris, B. (2003). Fatigue in composites, Cambridge, England: Woodhead Publishing Limited.

24. Szymiczek, M., Rojek, M. \& Wróbel, G. (2011).Test of composite pipe in the aspect of diagnostic of aging - fatigue changes. Proceedings of International Seminar on Science and Education, 16-23 April 2011(pp. 61-63). Rome, Italy.

25. Stabik, J., Szymiczek, M., Wróbel, G. \& Rojek, M. (2012). New stand for composite pipes testing. Proceedings of X International Conference of the Quality, Reliability and Long Usage of Technical Systems and Technological Processes, 20-27 November 2012 (pp. 63-66), Eilat, Israel.

26. Szymiczek, M., Wróbel, G., M. Rojek, M. \& Czapla T. (2013). Simulation diagnostics of the polyester-glass pipes degradation process; experimental basis, J. Achievements Mater. Manufact. Engineer., 59/1, 37-47.

27. Standards Association of Poland (2002). Poland standard: Plastics piping - Glass-reinforced thermosetting plastics (GRP) pipes - Determination of the apparent initial circumferential tensile strength. PN-EN 1394-2002.

28. Standards Association of Poland (1993). Poland standard: Plastics - Determination of flexural properties. PN-EN ISO 178-1993. 